\title{
Biodiversity Research on Coral Reef and Island Ecosystems: Scientific Cooperation in the Pacific Region ${ }^{1}$
}

\author{
Makoto Tsuchiya, ${ }^{2}$ René Galzin, ${ }^{3}$ and Neil Davies ${ }^{4}$
}

An INTERNATIONAL symposium entitled "Tropical Island Ecosystems and Sustainable Development" was held during 2-7 December 2006 in cooperation with three institutions: 21st Century COE (Center of Excellence) Program of University of the Ryukyus, Okinawa, Japan (UR), Centre de Recherches Insulaires et Observatoire de l'Environment, French Polynesia (CRIOBE), and Richard B. Gump South Pacific Research Station of University of California, Berkeley, French Polynesia (GUMP).

The event was timely for the Pacific region, where natural environments, including coral reef and island ecosystems, are increasingly disturbed by human activities. Cooperative science-based projects are urgently required for the conservation and sustainable use of insular ecosystems. Global climate change is having its most dramatic and immediate impact on polar regions and low-lying tropical islands. In the Pacific, phenomena such as increasing ocean temperatures and rising sea levels have impacts on coral reef ecosystems that are undoubtedly serious but remain insufficiently understood. Coral bleaching is probably one of the gravest consequences of climate change, with current bleaching events reported occurring widely with destructive results for coral reef

\footnotetext{
${ }^{1}$ Manuscript accepted 1 July 2007.

${ }^{2}$ Faculty of Science, University of the Ryukyus, Nishihara, Okinawa 903-0213, Japan (e-mail: tsuchiya@ sci.u-ryukyu.ac.jp).

${ }^{3}$ Centre de Recherches Insulaires et Observatoire de l'Environment, UMS 2978 CNRS-EPHE, BP 1013, 98729 Papetoai, Moorea, French Polynesia.

${ }^{4}$ Richard B. Gump South Pacific Research Station, University of California, Berkeley, BP 244, 98728 Moorea, French Polynesia (http://moorea.berkeley.edu).
}

Pacific Science (2008), vol. 62, no. 3:299-301 (C) 2008 by University of Hawai'i Press

All rights reserved ecosystems. In addition to rising temperatures, global carbon dioxide emissions and the potential acidification of oceans could add to the devastation of coral reefs. Furthermore, coral diseases have been reported recently in many reefs, and these might also be related to the current environmental deterioration. More research is needed to understand the local impact of global changes on small island ecosystems; only then can effective approaches be developed to protect and restore degraded habitats. There is no time for delay if coral reefs are to recover their healthy condition and to maintain the ecosystem function and services on which so many Pacific societies depend.

Another worldwide trend that has particularly important impacts on island ecosystems is the rapid increase in trade and travel (globalization), which spreads species to new geographic locations. Islands, which by definition evolved in relative isolation, are particularly threatened by invasive species. The most dramatic and tragic effects of biological invasions are already well documented for terrestrial island ecosystems; however, what makes some species more damaging and some systems more resistant or resilient to invasions is unclear. Islands represent a unique opportunity (a natural laboratory) to address this issue; they are also the places where its resolution is of far more than academic interest.

In addition to the impacts of globalization and climate change, there are more direct threats associated with increasing human population pressure on natural resources. Over the last 100 years, the human population has doubled worldwide and tripled in Japan. This explosive rate of growth far exceeds that of the past $10-15$ centuries and has severely taxed global resources. For example, to meet food demands, the fishing industry has harvested larger catches, ignoring the rules and balances of nature, and resulting in 
overfishing and great disturbance to fishery grounds. Human activities on land have degraded terrestrial ecosystems through habitat destruction. Consequences include not only the irreversible loss of endemic species from "biodiversity hotspots" but also the erosion of human cultural diversity (which is often tightly linked to its unique natural heritage). Unsustainable terrestrial development also threatens marine systems through large inflows of exogenous materials and fine particles to coastal zones and the eutrophication of some reefs. Such conditions have allowed increased population growth of algal species, which has also tipped coral reef systems toward a new ecological equilibrium that does not favor human well-being. Most seriously and devastatingly, some coral reefs have become the targets of "reclamation" projects and have disappeared completely.

Sustainable use for coral reef and island ecosystems must be the most important and urgent challenge facing the tropical and subtropical Pacific regions. It is obvious that solutions must involve a much greater understanding of biodiversity and its relationship to human society; more cooperative research in the Pacific region is an urgent priority.

In Japan, the 21st Century COE Program has been promoted by the Ministry of Education, Culture, Sports, Science, and Technology since 2002 to establish global research and education centers based on the principle of competition and thus to create internationally competitive universities of the highest quality in the world. This program is expected to foster young researchers as well as to promote university education reform. Based on these fundamental principles, the 21st Century COE Program of UR aims to provide comprehensive analysis of biodiversity in coral reefs and islands and to establish the university as an international center for research and education concerning these issues. Through the COE program, UR is making every effort to accomplish this goal by research and education rooted in unique characteristics of natural environments in Okinawa.

The COE program regards the marine and terrestrial environment of subtropical islands including coral reefs as a single large ecosystem in analyzing the dynamics and evolution of biodiversity in the Ryukyu archipelago. Researchers from several fields collaborate in research and education regarding the evolution and the mechanism of retention of biodiversity in the Ryukyu Islands, employing methodologies from various fields of study, from molecular biology to ecology, and examining diverse phyla from microorganisms to higher plants and vertebrates, and from land to the ocean. The COE program aims to establish an international research and education center in the Asia Pacific region to encourage young researchers. This center would contribute to a network of cooperative programs in the Pacific that fosters the training of young scientists and provides crucial advances in our understanding of coral reef and island ecosystems in the tropical and subtropical Pacific region.

Toward these broad goals, more than 50 international experts met for 4 days of discussion on the island of Moorea, a model ecosystem embedded in the natural laboratory of French Polynesia. With its international research stations (France and the United States), Moorea represents an important hub of the Pacific research network. Over half the scientists attending the meeting came from Okinawa, and one goal of the conference was to develop collaboration among French Polynesia, other Pacific regions, and Japan. The conference emphasized that sustainable development can only be achieved through a much deeper understanding of how physical, biological, and social processes interact in tropical systems. Terrestrial and marine systems are interconnected, and integrated approaches are essential. Islands demonstrate this clearly and are excellent model systems for sustainable development. Knowledge creation through research is the necessary foundation, but science alone is not sufficient for sustainable development. We must also create institutions to ensure the effective transfer of knowledge and technologies to government agencies, nongovernment organizations, local communities, and the private sector (applied science and science-based management). Formal education and training at all levels is thus 
crucial, as well as raising public awareness through informal educational initiatives. The Japanese and Polynesia-based scientists agreed to work together toward these goals and are now beginning to develop specific research and educational programs, in particular:

(1) Graduate training course-The COE of UR has a 2-month summer program for $\mathrm{Ph} . \mathrm{D}$. students in tropical ecosystems held in Okinawa every year. We are considering the possibility of jointly holding this Ph.D. training program in Moorea in the near future. The organization and topics of the program are being discussed. It would involve international professors and students.

(2) Graduate research collaborations-We will encourage graduate students from our institutions to carry out research in laboratories in both French Polynesia and Okinawa. These exchanges will include collaboration of their professors and the research themes identified by the conference.

(3) Advanced training program-We aim to create courses (perhaps based on the experience of the Ph.D.-level program) targeting natural resource managers from the Pacific islands.

The symposium also provided the occasion to launch a new initiative in French Polynesia: GUMP, CRIOBE, and French Polynesia agreed to begin working together, and eventually with other partners, toward a "Moorea Ecostation." The goal of the ecostation is to create a platform to further develop the Moorea model ecosystem and to support sustainable development throughout French Polynesia. The initiative will further research, education, and training programs building on the existing infrastructure of GUMP and CRIOBE. The Moorea Ecostation seeks to establish partnerships with similar research hubs throughout the region. COE of UR has begun this cooperative endeavor. New partners will be welcome.

Acknowledgments: Thanks are due to Dieter Mueller Dombois, Professor Emeritus of University of Hawai' $i$, who gave an important keynote lecture at the symposium. We thank the Coral Reef Initiative for the South Pacific (CRISP), Moorea Dolphin Center, L'Initiative Française sur les Récifs Coralliens (IFRECOR), and Air Tahiti for their wonderful cooperation. We are also grateful to Haut-Commissariat de la Republique en Polynesie Française for their kind hospitality. The COE members from Okinawa especially wish to thank all members of CRIOBE and GUMP for their assistance and kind hospitality during the stay in French Polynesia.

Curt Daehler, editor of Pacific Science, kindly gave us the opportunity to publish the results of our symposium in this special issue of Pacific Science. The publication of this special issue is supported by a grant for the 21st Century COE Program "The Comprehensive Analyses on Biodiversity in Coral Reef and Island Ecosystems in Asian and Pacific Regions" from the Ministry of Education, Culture, Sports, Science, and Technology, Japan (Monbukagakusho). 
